\title{
Characterization of Vestibular Dysfunction in the Mouse Model for Usher Syndrome 1F
}

\author{
Kumar N. Alagramam, ${ }^{1}{ }_{4}$ ohn S. Stahl, ${ }^{2}$ Sherri M. Jones, ${ }^{3}$ Karen S. Pawlowski, ${ }^{4}$ \\ AND Charles G. WRight \\ ${ }^{1}$ Department of Otolaryngology—Head and Neck Surgery, University Hospitals of Cleveland, Case Western Reserve University, \\ 11100 Euclid Avenue, Cleveland, OH 44106, USA \\ ${ }^{2}$ Department of Neurology, University Hospitals of Cleveland, Case Western Reserve University, Cleveland, OH 44106, USA \\ ${ }^{3}$ Department of Communication Sciences and Disorders, East Carolina University, Greenville, NC 27858, USA \\ ${ }^{4}$ Department of Otolaryngology—Head and Neck Surgery, Southwestern Medical Center, Dallas, TX 75235, USA
}

Received: 14 June 2004; Accepted: 3 December 2004; Online publication: 10 June 2005

\begin{abstract}
The deaf-circling Ames waltzer (av) mouse harbors a mutation in the protocadherin 15 (Pcdh15) gene and is a model for inner ear defects associated with Usher syndrome type 1F. Earlier studies showed altered cochlear hair cell morphology in young $a v$ mice. In contrast, no structural abnormality consistent with significant vestibular dysfunction in young av mice was observed. Light and scanning electron microscopic studies showed that vestibular hair cells from presumptive null alleles $P c d h 15^{a v-T g}$ and $P c d h 15^{a v-3 J}$ are morphologically similar to vestibular sensory cells from control littermates, suggesting that the observed phenotype in these alleles might be a result of a central, rather than peripheral, defect. In the present study, a combination of physiologic and anatomic methods was used to more thoroughly investigate the source of vestibular dysfunction in Ames waltzer mice. Analysis of vestibular evoked potentials and angular vestibulo-ocular reflexes revealed a lack of physiologic response to linear and angular acceleratory stimuli in Pcdh15 mutant mice. Optokinetic reflex function was diminished but still present in the mutant animals, suggesting that the defect is primarily peripheral in nature. These findings indicate that the mutation in Pcdh15 results in either a functional abnormality in the vestibular receptor organs or that the defects are limited to the vestibular nerve.
\end{abstract}

Correspondence to: Kumar N. Alagramam - Department of Otolaryngology-Head and Neck Surgery, University Hospitals of Cleveland - Case Western Reserve University - 11100 Euclid Avenue, Cleveland, OH 44106, USA. Telephone: (216) 844-7261; email: kna3@cwru.edu
AM1-43 dye uptake has been shown to correlate with normal transduction function in hair cells. Dye uptake was found to be dramatically reduced in Pcdh15 mutants compared to control littermates, suggesting that the mutation affects hair cell function, although structural abnormalities consistent with significant vestibular dysfunction are not apparent by light and scanning electron microscopy in the vestibular neuroepithelia of young animals.

Keywords: deafness, vestibular defects, Ames waltzer, protocadherin 15 (Pcdh15), VsEP, aVOR

\section{INTRODUCTION}

The Ames waltzer mouse (av) carries a recessive mutation, which causes deafness and balance disturbance. The gene linked to the $a v$ mutation was identified to be a protocadherin, Pcdh15 (Alagramam et al. 2001a). Mutations in the human homologue of Pcdh15 were identified in several families, and, in many cases, mutations segregate with the disease phenotype and generate premature stop codons (Alagramam et al. 2001b; Ahmed et al. 2001). Mutations in the Pcdh15 $15^{a v-3 J}$ and Pcdh15 $5^{a v-T g}$ alleles of $a v$ also result in premature truncation of Pcdh15 transcript (Alagramam et al. 2001a,b). More recently, it has been shown that mutation in $\mathrm{PCDH} 15$ is associated with nonsyndromic deafness DFNB23 (Ahmed et al. 2003). It has also been shown that R245X mutation of PCDH15 may be the most 
common cause of Usher syndrome type $1 \mathrm{~F}$ (USH1F) in the Ashkenazi Jewish population (Ben-Yosef et al. 2003). Therefore $a v$ would appear to be a valuable model for better understanding inner ear pathology in USH1F and DFNB23 patients.

The $a v$ mouse mutation was first reported in 1961 (Schaible 1961), and, since then, there have been several reports of inner ear pathology in av mutants, although most reports have focused on cochlear abnormalities (Osako and Hilding 1971; Alagramam et al. 1999, 2000; Raphael et al. 2001). Osako and Hilding studied the physiology of $a v$ mice (presumed to be the original allele of $a v$ ) and found no evidence of auditory function at any time during postnatal development or in the adult. These findings are consistent with the degenerative structural changes in stereocilia and hair cells of the cochlea seen as early as P15. Although the $a v$ mice displayed circling behavior, Osako and Hilding found no anatomical defects in the vestibular apparatus. Transmission electron microscopic study of the saccular neuroepithelium in "adult" (age not specified) av mice failed to reveal any ultrastructural abnormalities in the sensory cells, supporting cells, or neural elements (Osako and Hilding 1971). Therefore the structural basis for the early-onset circling behavior in av mice is not clear.

Preliminary data from the $P c d h 15^{a v-T g}$ allele suggested that pathology of the saccule in the vestibular periphery might contribute to the circling behavior (Alagramam et al. 1999, 2000). In adult mutants, 50 days after birth (P50) or older, the neuroepithelium of the saccular macula was found to be completely absent, with no recognizable hair cells or supporting cells. The neuroepithelia of the utricle and cristae of the semicircular canals appeared normal in light microscopic cross sections in the Pcdh15 $5^{a v-T g}$ allele. The loss of saccular neuroepithelium was described as being consistent with the compromised balance function observed in this mutant; however, subsequent study of inner ear cross sections showed that the vestibular end organs of young adult mutants ( P20-P30) appeared normal compared to control littermates, although the mutant mice displayed circling behavior as early as P10 (unpublished data). Indeed, the functional status of the vestibular system in Pcdh15 mutants has not been evaluated up to the present time. This study used several approaches to characterize the vestibular phenotype in $a v$ mice and included a more detailed anatomic study of the vestibular periphery.

\section{MATERIALS AND METHODS}

Alleles of $a v$ used in this report

Mutation in different alleles of $a v$ used in this report including Pcdh15 $5^{a v-T g N 2742 R p w}$, Pcdh15 $5^{a v-3 J}, P c d h 15^{a v-2 J}$, and $P c d h 15^{a v-J}$ was reported earlier (Alagramam et al. 2001a,b). Pcdh15 $5^{a v-T g N 2742 R p w w}$ is referred to as $P c d h 15^{a v-T g}$ in this report. Like other protocadherins, the wild-type Pcdh15 is predicted to be a transmembrane protein. Mutations Pcdh $15^{a v-J}$ and $P c d h 15^{a v-2 J}$ are considered to be less deleterious because these alleles carry an inframe deletion leading to absence of a small number of amino acids in the extracellular domain of the protein. In contrast, $P c d h 15^{a v-T g}$ and $P c d h 15^{a v-3 J}$ alleles are presumed to be functional null alleles because mutation in these alleles is predicted to code for Pcdh15 protein lacking the entire transmembrane domain and all of the cytoplasmic domains. We focused most of our work on the $P c d h 15^{a v-T g}$ allele; earlier studies have shown that mutations in the Pcdh $15^{a v-T g}$ allele and the Pcdh $15^{a v-3 J}$ allele are likely to have the same effect on the inner ear phenotype. Data from the Pcdh15 $5^{a v-3 J}$ (C57BL/6J background) are also included to show that the genetic background does not have any effect on the inner ear phenotype in young mice.

\section{Morphology}

Histological preparation for light microscopy was performed using standard procedures described previously (Alagramam et al. 1999). For histological studies of inner ear morphology in adult animals (P30-P50, 1 year), cochlear tissues were fixed by perilymphatic perfusion of $2.5 \%$ phosphate-buffered glutaraldehyde. Following fixation, specimens were rinsed in buffer, decalcified in 0.35 M EDTA, and embedded in glycol methacrylate. Tissue sections were then cut at a thickness of $2-5 \mu \mathrm{m}$ and stained with toluidine blue for light microscopic study. For scanning electron microscopic studies, inner ears from ten mutant mice and five wild-type controls were used for every allele examined at each of four postnatal time points: P2, P5, P10, and P20. These time points were used to study possible morphological changes during the period of postnatal maturation of the inner ear. Findings from the SEM studies were correlated with observations from light microscopic examination of plastic embedded inner ear cross sections.

Inner ears to be studied by SEM were fixed by perilymphatic perfusion of $2.5 \%$ glutaraldehyde as described previously (Alagramam et al. 2000). The temporal bones were opened to permit removal of the otoconial membranes from the neuroepithelia of the saccule and utricle, and the membranous labyrinths were stained with $1 \%$ osmium tetroxide. Tissue specimens including the cristae of the semicircular ducts and the maculae of the saccule and utricle were microdissected from the inner ears, dehydrated in ethanol, and critical point dried. The specimens were mounted on aluminum stubs using colloidal silver 
adhesive and sputter coated with gold palladium before study using a JEOL 848 scanning electron microscope.

\section{Vestibular evoked potential}

Vestibular neural function was assessed with linear vestibular evoked potentials (VsEPs). Linear VsEPs are compound action potentials of the vestibular portion of the eighth cranial nerve and its central relays and are generated by the utricle and saccule in response to linear acceleration stimuli.

Four alleles of Ames waltzer and age-matched heterozygote controls were evaluated: Pcdh $15^{a v-J}$ (ten mutants, seven controls), Pcdh $15^{a v-2 J}$ (three mutants, two controls), Pcdh15 ${ }^{a v-T g}$ (ten mutants, five controls), and $P c d h 15^{a v-3 J}$ (ten mutants, five controls); results from $P c d h 15^{a v-T g}$ and $P c d h 15^{a v-3 J}$ were identical. Table 1 lists the average age of each group of mice at the time of testing. Swimming behavior was recorded using a digital camera. Each mouse was placed in a glass aquarium filled with tepid water. Normal mice come to the surface of the water immediately and maintain horizontal bodyline swimming at the surface. Lack of swimming ability is observed when the mouse remains submerged or submerges often, completely disoriented, turning and tumbling beneath the surface.

The methods for recording linear VsEPs in mice have been reported in detail elsewhere (Jones and Jones 1999; Jones et al. 1999, 2002). Briefly, animals were anesthetized (Equithesin, $4 \mu \mathrm{l} / \mathrm{g}$, intraperitoneal; Lidocaine 1\%, $100 \mu \mathrm{l}$, subcutaneously at the scalp), and the skull was prepared with a head mount. Skull anchor screws were placed near the coronal and lambdoidal sutures and a stainless-steel electrode placed epidurally just posterior to the lambdoidal suture. A thumbscrew was secured at the midline, and all was encased with fast-set plaster. Two additional electrodes were placed subcutaneously behind the left and right pinnae (inverting electrodes) and a third at the ventral neck (ground). The animal was placed supine, and the head was secured to a custom platform attached to an electromechanical shaker with the naso-occipital axis oriented vertically.

Stimuli were linear acceleration pulses, 2-ms duration, nine pulses per second, presented in two directions, normal and inverted. Normal polarity was defined as upward displacement of the shaker platform, whereas inverted was downward platform displacement. Stimulus amplitude was measured in jerk $\left(\mathrm{d} a / \mathrm{d} t\right.$, i.e., $\mathrm{g} / \mathrm{ms}$, where $1.0 \mathrm{~g}=9.8 \mathrm{~m} / \mathrm{s}^{2}$ and $1.0 \mathrm{~g} /$ $\mathrm{ms}=9.8 \mu \mathrm{m} / \mathrm{ms}^{3}$ ) using a calibrated accelerometer attached to the shaker platform (Jones et al. 2002). The output of the accelerometer was differentiated electronically, and stimulus amplitude was described in $\mathrm{dB}$ re: $1.0 \mathrm{~g} / \mathrm{m} \mathrm{s}$. Stimulus amplitude ranged from -18 to $+6 \mathrm{~dB}$ re: $1.0 \mathrm{~g} / \mathrm{ms}$, adjusted in 3-dB steps.

Two-channel signal averaging was used to resolve vestibular responses from background electroencephalographic activity. Electrophysiological activity was amplified $(200,000 \times)$, filtered $(300-3000 \mathrm{~Hz})$, and VsEPs were recorded to normal and inverted stimulus polarities (1024 points, $10 \mu$ s per point, 128 responses per averaged waveform). Four waveforms were obtained for each stimulus intensity, two for normal polarity stimuli and two for the inverted polarity. Summing the responses to normal and inverted polarities and dividing the result by 2 was completed offline and produced the final averaged waveforms used for analysis. Therefore two final waveforms were obtained for each stimulus amplitude.

Three response parameters were quantified: threshold, peak latencies, and peak-to-peak amplitudes. Threshold is a measure of the sensitivity of the gravity receptor end organs. Threshold, measured in $\mathrm{dB}$ re: 1.0 $\mathrm{g} / \mathrm{ms}$, was defined as the stimulus amplitude midway between that which produced a discernible VsEP and that which failed to produce a response. Peak latency provides a measure of the neural timing of synaptic events and conduction along the vestibular pathways. Peak latencies, measured in microseconds $(\mu \mathrm{s})$, were

TABLE 1

\begin{tabular}{|c|c|c|c|c|c|c|c|}
\hline \multicolumn{8}{|c|}{ Average data for VsEP thresholds, latencies, and amplitudes of heterozygote mice } \\
\hline Strain & Age (days) & Threshold $\left(d B_{\text {re: } 1.0 \mathrm{~g} / \mathrm{ms}}\right)$ & $P 1(\mu s)$ & $P 2(\mu s)$ & $P 3(\mu s)$ & $P 1 / N 1(\mu V)$ & $P 2 / \mathrm{N} 2(\mu \mathrm{V})$ \\
\hline$+/ J$ & $49 \pm 6$ & $-8.4 \pm 3.8$ & $1289 \pm 80$ & $2104 \pm 103$ & $3055 \pm 293$ & $3.4 \pm 0.8$ & $2.6 \pm 0.9$ \\
\hline$J / J$ & $47 \pm 5$ & NR & NR & NR & NR & NR & NR \\
\hline$+/ 2 J$ & $40 \pm 3$ & $-10.5 \pm 4.2$ & $1435 \pm 113$ & $2267 \pm 11$ & $3185^{\mathrm{a}}$ & $3.9 \pm 2.9$ & $3.6 \pm 2.7$ \\
\hline $2 J / 2 J$ & $39 \pm 3$ & $-4.5^{\mathrm{a}}$ & $1230^{\mathrm{a}}$ & $2035^{\mathrm{a}}$ & NP & $1.8^{\mathrm{a}}$ & $0.4^{\mathrm{a}}$ \\
\hline$+/ T g$ & $70 \pm 1$ & $-10.5 \pm 3.0$ & $1260 \pm 31$ & $2025 \pm 88$ & $2893 \pm 188$ & $3.7 \pm 0.9$ & $3.2 \pm 2.0$ \\
\hline$T g / T g^{\mathrm{b}}$ & $69 \pm 1$ & NR & NR & NR & NR & NR & NR \\
\hline
\end{tabular}

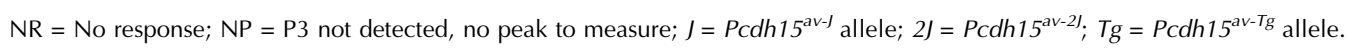

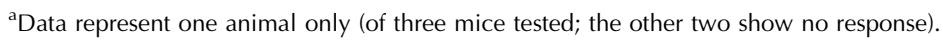

${ }^{\mathrm{b}}$ Results from $\mathrm{Tg}$ allele and av3J were identical. 
obtained by measuring the time from stimulus onset to the occurrence of the first three response peaks (P1, P2, and P3). Peak to peak amplitudes, measured in microvolts $(\mu \mathrm{V})$, provide an estimate of neural synchrony and the population of cells contributing to the response. Amplitudes were obtained by subtracting the amplitude of each positive peak from its respective negative peak. Thresholds, latencies, and amplitudes were averaged for each group.

\section{Angular vestibulo-ocular reflex and optokinetic reflex}

The integrity of circuits mediating the angular vestibulo-ocular reflex (aVOR) was assessed by recording eye movements in response to sinusoidal and step rotations of the animal about an earth-vertical axis, as well as in response to constant velocity rotations of the visual surround about the same vertical axis (optokinetic reflex, OKR). Eye movement recordings were obtained in six animals, aged approximately 150 days, and homozygous for the Pcdh $15^{a v-3 J}$ mutation, using techniques previously described (Stahl 2002, 2004; Stahl et al. 2000). Briefly, animals were prepared several days prior to the first recordings by surgically implanting an acrylic head fixation pedestal under halothane anesthesia. The pedestal was placed at an angle such that during recordings, the animal's head would be fixed in a natural position in which a line drawn from the inner to the outer canthus would be in, or pitched slightly down from, the earth-horizontal plane. Prior to each recording session, the recorded eye was pretreated with $0.5 \%$ physostigmine ophthalmic drops to limit pupil dilation in darkness. During recordings, the animal's body was loosely restrained in an acrylic tube with the pedestal securely bolted to an extension of the tube. The assembly was mounted on a turntable and completely enclosed in a motorized drum painted with a highly contrasted pattern. Vestibular or optokinetic stimulation about an earth-vertical axis was accomplished by computercontrolled rotation of the table or drum motors, respectively. A video camera fixed to the table provided a continuous picture of one eye under infrared illumination. Commercial video oculography hardware/software (ISCAN, Burlington, MA) was used to track the position of the pupil centroid and a reference corneal reflection at a rate of 60 samples/s. These positions, output as analog voltages by the ISCAN system, were then low-pass filtered at $100 \mathrm{~Hz}$ to prevent subsequent aliasing and acquired to another computer along with the table and drum velocities at a rate of 200 samples/s. The $\mathrm{X}-\mathrm{Y}$ positions of the pupil and reference reflection were subsequently converted to angular eye-in-head position using a geometric algorithm as previously described (Stahl 2002; Stahl et al. 2000).

The aVOR was tested in four Pcdh $15^{a v-3 J}$ mutants aged approximately 150 days and compared to normative data generated from $11 \mathrm{C} 57 \mathrm{BL} / 6 \mathrm{~J}$ (the background strain on which the $P c d h 15^{a v-3 J}$ mutation is maintained) animals aged 57-175 days. The aVOR was probed using sinusoidal rotation in the light and dark at the following frequencies and nominal amplitudes: $0.1 \mathrm{~Hz}, 10^{\circ}(0$-peak $)$ amplitudes; $0.2 \mathrm{~Hz}, 10^{\circ}$; $0.4 \mathrm{~Hz}, 10^{\circ} ; 0.8 \mathrm{~Hz}, 9.2^{\circ} ; 0.8 \mathrm{~Hz}, 4.6^{\circ}$; and $1.6 \mathrm{~Hz}, 3.9^{\circ}$. Each data record captured approximately $50 \mathrm{~s}$ of stimulus and response, and in each session, we acquired two to three records for each stimulus condition. Responses were quantified in terms of gain and phase. Gain is the ratio of the amplitudes of the eye velocity and the head velocity, where a value of 1.0 would signify an eye movement that perfectly compensated for the amplitude of the head movement. Phase is a measure of the relative timing of the sinusoidal eye and head velocities, where a value of $0^{\circ}$ would signify that the peak velocity of the sinusoidal eye movement was perfectly aligned in time with the peak velocity of the head movement. Gain and phase were extracted in the conventional fashion: table (head) velocity and eyein-head velocity were calculated by taking the derivatives of the respective angular position signals. Fast phases of vestibular nystagmus were identified using velocity criteria and patched by interpolation from the surrounding record, using an automated algorithm supplemented by manual review (Stahl et al. 2000). Records were then divided into individual cycles and pointwise averaged to generate a single average cycle of eye velocity and head velocity. The amplitude and phase of these averaged curves were then extracted by Fourier decomposition. Finally, gain was calculated as the ratio of the amplitude of the eye and table velocities [i.e., Gain $\left.=\operatorname{mag}\left(E^{\prime}\right) / \operatorname{mag}\left(H^{\prime}\right)\right]$, and phase was calculated as the difference of their phases [i.e., Phase $=\arg \left(E^{\prime}\right)-$ $\arg \left(H^{\prime}\right)$ ]. Gain and phase values were calculated for each record (trial) and averaged to generate single gain and phase versus stimulus frequency curves for each animal's session. Curves from two sessions were averaged for each animal to generate single gain and phase curves for each animal, and finally, the curves from the six animals were used to generate average curves and standard deviations for the mutant genotype.

In addition to the sinusoidal stimuli, the aVOR was also probed using a higher-acceleration, step-like stimulus (amplitude 15, peak velocity 105/s, repetition rate $0.25 \mathrm{~Hz}$ ), delivered in darkness. Animals were tested in the standard position, as well as with the head and body pitched $40^{\circ}$ nose-down from the standard position. Responses were measured by position gain, calculated as the peak-to-peak change 
of horizontal angular eye position divided by the change in angular position of the table over the same period. As in the case of the response to sinusoidal rotations, a gain value of 1.0 would signify that the eye rotation compensated perfectly for the amplitude of the head rotation. For each animal, gains were calculated separately for rightward- and leftwarddirected rotations, and then these were averaged to generate a single gain for each animal. The singleanimal gains were then averaged to generate a single value for each genotype. Response was compared to a group of six C57BL/6 animals aged 56-92 days.

The OKR was tested in six mutants aged 151-197 days and compared to the laboratory's reference curve for young adult mice, which was derived from a group of 20 C57BL/ 6 controls, aged 65-94 days. Each OKR record consisted of the response to a set of 4-s periods of constant velocity drum rotation at $\pm 2.5^{\circ} / \mathrm{s}, 5^{\circ} / \mathrm{s}$, $10^{\circ} / \mathrm{s}, 20^{\circ} / \mathrm{s}$, and $40^{\circ} / \mathrm{s}$, separated by 3.5 -s periods during which the drum accelerated to a stable velocity with the animal in darkness. For each period of constant velocity drum rotation in the light, fast phases were removed from the eye velocity record by interpolation and the average velocity for the period was calculated. Gain was then calculated as the ratio of the average eye and drum velocities. Three to four similar records were collected per session, and the gains at each stimulus velocity were averaged to generate a gain versus stimulus velocity curve for the session. Multiple sessions and animals were combined as with the aVOR analysis to generate average curves and standard deviations for the mutant and control genotypes.

\section{AM1-43 dye experiment}

Freshly dissected inner ear specimens from P10 heterozygous $\left(+/ P c d h 15^{a v-3 J}\right.$ or $\left.+/ P c d h 15^{a v-2 J}\right)$ and homo- zygous (Pcdh15 $5^{a v-3 J} / P c d h 15^{a v-3 J}$ or Pcdh15 $5^{a v-2 J} /$ $P c d h 15^{a v-2 J}$ ) mice were labeled by immersion in AM1-43 dye solution (20- $\mu \mathrm{M}$ dye solution prepared in water). The tissue was not precultured before labeling. To ensure that the dye had good access to the vestibular neuroepithelia, the vestibule was widely opened at the oval window, sacrificing the basal part of the cochlea, and a hole was made in the lateral semicircular canal wall. The inner ears were immersed in the dye for $10 \mathrm{~min}$ at room temperature (RT). The tissue was then washed with PBS four times (10 min each at RT) and fixed in $4 \%$ paraformaldehyde. Specimens were rinsed and epithelia dissected in PBS. The utricular and saccular maculae and the cristae of the semicircular ducts were mounted in glycerin and coverslipped. Slides were then viewed and photographed using a Zeiss KL 300 microscope with Texas red filter set.

\section{RESULTS}

\section{Morphology}

The vestibular sensory cells in mutants at P2, P5, P10, and P20 appeared morphologically normal, although the circling behavior is observed as early as P10. Sensory cell loss in the saccular macula becomes apparent in animals harboring the presumptive null mutation (either $P c d h 15^{a v-T g}$ or $P c d h 15^{a v-3 J}$ ) by P30 to P40 and progresses to total loss of the saccular neuroepithelium in animals 7 months to 1 year old (Fig. 1). Surprisingly, no lesions in the cristae or utricular macula or their corresponding nerve fibers were found by light microscopy even in 1-year-old mutant mice. Pcdh $15^{a v-3 J}$ mutants showed the same

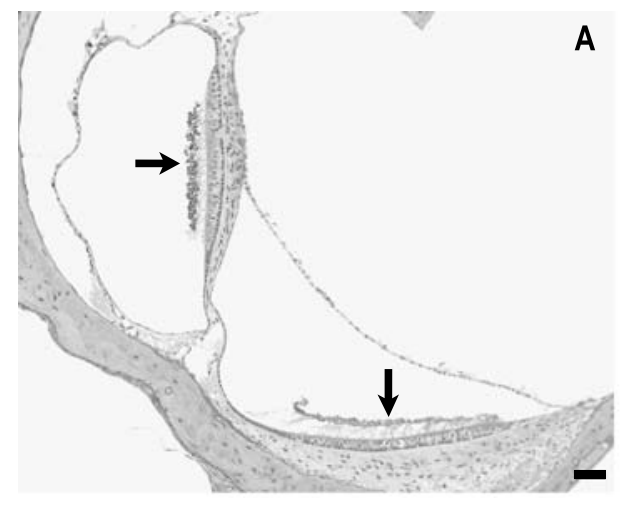

FIG. 1. Cross sections of the vestibule from a control animal (A) and a $P c d h / 5^{\text {av-Tg }}$ mutant (B) at 1 year of age. In both micrographs, the arrow at lower right indicates the area of the saccular macula, and the arrow at upper left indicates the utricular macula. In the mutant, the saccular neuroepithelium has completely degenerated,

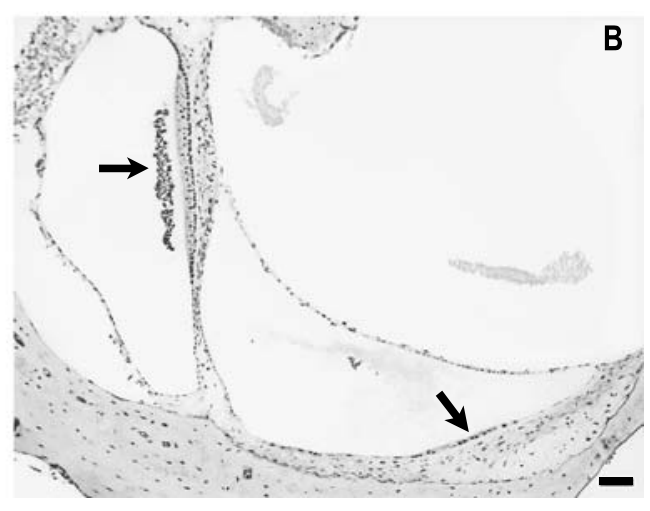

whereas in the control, the saccular macula is normal. The utricular macula is of normal configuration in both the mutant and the control. Toluidine blue stain. The scale bar at lower right indicates $50 \mu \mathrm{m}$ in both micrographs. 
phenotype as $P c d h 15^{a v-T g}$ at all stages that we examined (data not shown).

Degeneration of saccular otoconia can be seen by both light microscopy and SEM starting at about 25 days after birth in Pcdh15 $5^{a v-T g}$ mutants. In these animals, the crystalline layer of the saccular otoconial membrane becomes thinned out, and the crystals appear to undergo demineralization and break into small fragments (Fig. 2).

SEM and light microscopic study of the vestibular neuroepithelia from $P c d h 15^{a v-T g}$ or $P c d h 15^{a v-3 J}$ mice at P2, P10, and P20 showed that the saccular, utricular, and ampullary receptors were not significantly different from those of normal siblings. Of particular interest is P10: vigorous circling and head bobbing behavior is observed as early as P10 in the av mutants, and we expected to see structural changes in the vestibular hair cells at P10, if not earlier. After examining several samples at each time point $(n=$ 10), we failed to find an observable anatomical correlate that would readily explain the severe vestibular dysfunction observed in the $P c d h 15^{a v-3 j}$ mice at P10 (Figs. 3 and 4). Subsequent work on $P c d h 15^{a v-J}$ and $P c d h 15^{a v-2 J}$ showed similar results (data not shown).
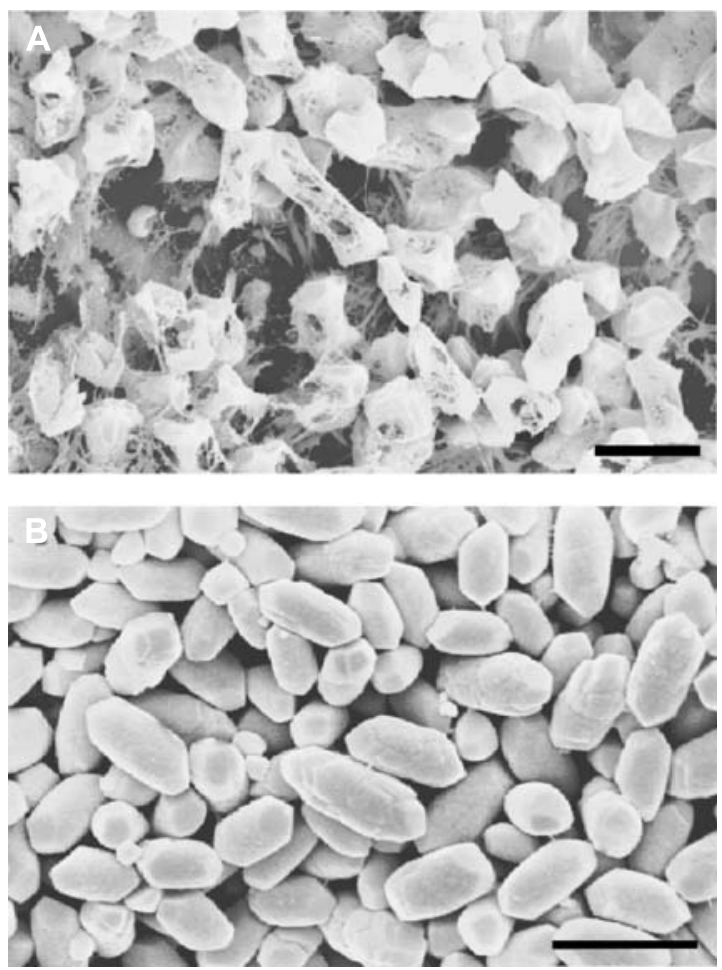

FIG. 2. Degeneration of saccular otoconia in the $P c d h 15^{a v-T g}$ mutant as seen by scanning electron microscopy. A. Crystalline layer of the saccular otoconial membrane from a 30-day-old mutant showing fragmented and demineralized otoconial crystals. B. Normal otoconia from the saccule of a young-adult control mouse. The scale bars at lower right in both micrographs indicate $5 \mu \mathrm{m}$.

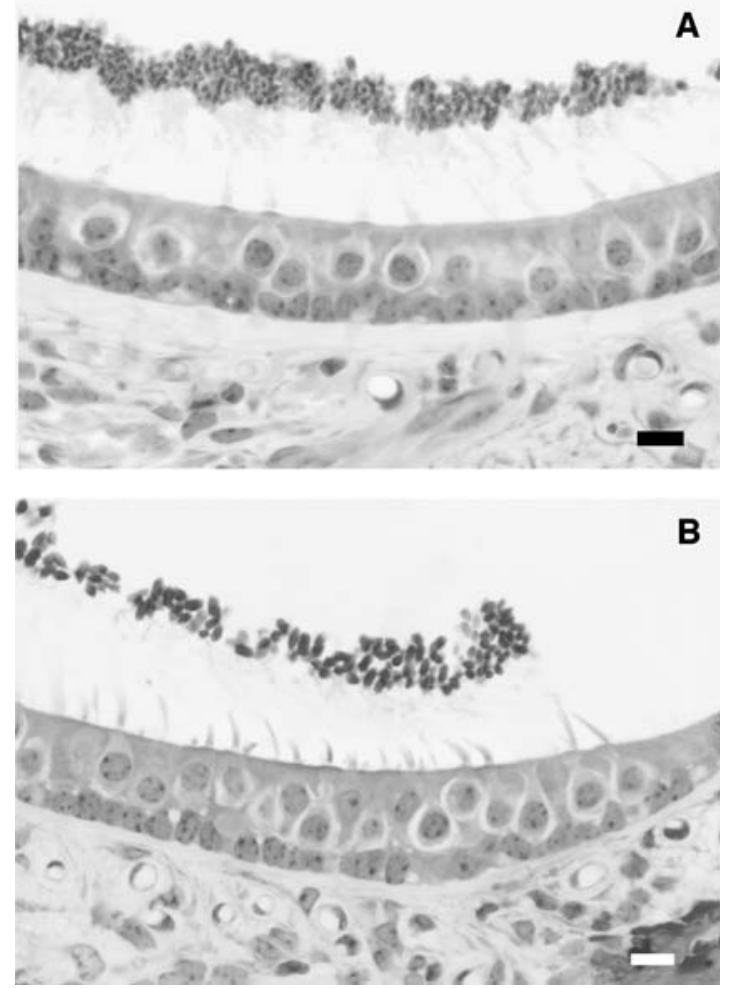

FIG. 3. Cross sections of the saccular macula from a control

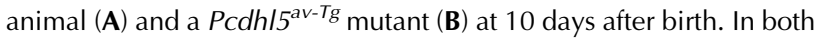
cases, the saccular neuroepithelium is composed of normal-appearing sensory and supporting cells. Toluidine blue stain. The scale bar at lower right indicates $10 \mu \mathrm{m}$ in both micrographs.

\section{VsEP}

All heterozygote controls had VsEPs similar to data previously published for normal mice (Jones and Jones 1999; Jones et al. 1999, 2002). VsEP thresholds and amplitudes were indistinguishable among the heterozygote groups (Table 1). Peak latencies, however, showed some differences. On average, peak latencies were longest for the $P c d h 15^{a v-2 J}$ heterozygotes and shortest for the $P c d h 15^{a v-T g}$ heterozygotes (Fig. 5). Interestingly, the $P c d h 15^{a v-2 J}$ heterozygotes were the youngest of the mice tested, whereas the Pcdh15 $5^{a v-T g}$ heterozygotes were the oldest (Table 1).

Most homozygotes were unable to swim. They were disoriented and dove beneath the water's surface. The Pcdh15 $5^{a v-T g}$ mice could not find the surface of the water and had to be rescued. Three Pcdh $15^{a v-J}$ mice appeared to keep the nose above water but swam in pirouette fashion, spinning like a top in one location. Linear VsEPs were absent in all homozygotes that could not swim or swam abnormally (Fig. 5). In one Pcdh $15^{a v-2 J}$ mouse, VsEPs were present with response peak latencies within normal limits, but thresholds were elevated $(-4.5 \mathrm{~dB}$ re: $1.0 \mathrm{~g} / \mathrm{ms})$ and response peak amplitudes reduced in comparison to 

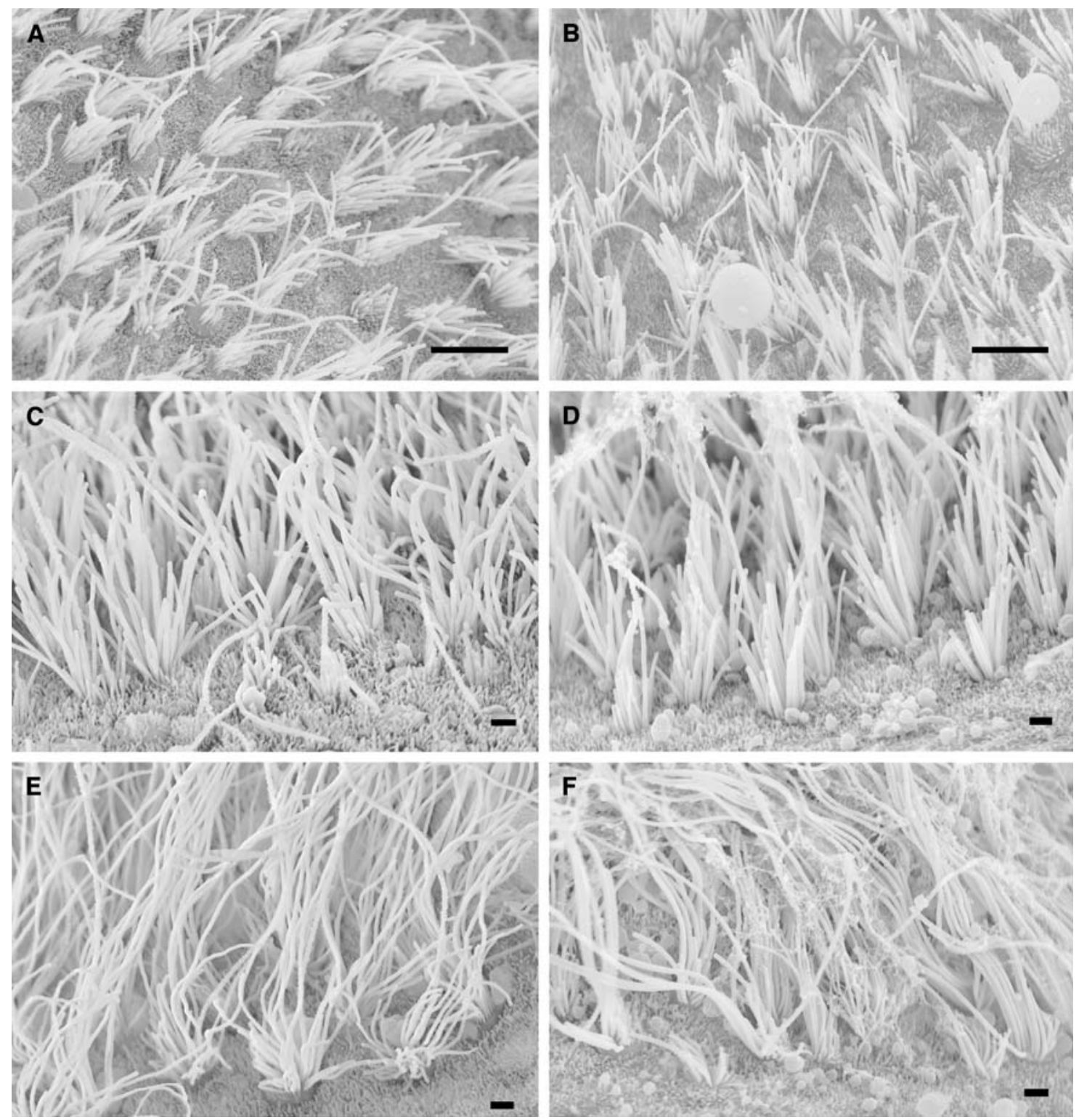

FIG. 4. Scanning electron micrographs showing stereocilia on vestibular sensory cells from control $(\mathbf{A}, \mathbf{C}, \mathbf{E})$ and $P c d h / 5^{\mathrm{av}-\mathrm{Tg}}$ mutant (B, D, F) inner ears at 10 days after birth. No dramatic differences in configuration of stereocilia are seen between control and mutant

controls (Fig. 6). Interestingly, this particular mouse displayed normal swimming behavior but did show circling behavior in the cage.

\section{aVOR}

Plots of aVOR gain and phase versus stimulus frequency are shown in Figure 7. Control animals rotated in the light-generated aVOR gains in excess of 0.8 at 0.1 $\mathrm{Hz}$, rising toward the theoretical ideal of 1.0 at the highest stimulus frequencies. Likewise, phase in the light remained close to the theoretical ideal of $0^{\circ}$ lead. In the dark, average gain rose steadily from around 0.3 at $0.1 \mathrm{~Hz}$ to approximately 0.8 at $1.6 \mathrm{~Hz}$, and the phase

animals at this age in either the saccule (top row), utricle (middle row), or superior crista (bottom row). The scale bar at lower right in $\mathbf{A}$ and $\mathbf{B}$ indicates $5 \mu \mathrm{m}$; scale bars in the other micrographs all equal $1 \mu \mathrm{m}$.

versus frequency curve rose from around $40^{\circ}$ lead to around $0^{\circ}$ lead. This gain and phase behavior of the aVOR in darkness reflects the biophysics of the semicircular canals and is qualitatively similar to that reported in all species studied to date (Wilson and Jones, 1979). In striking contrast to the normal controls, Pcdh $15^{a v-3 J}$ mice did not respond to rotation in darkness (gain of near zero at all tested stimulus frequencies). For rotation in the light, gain averaged approximately 0.6 and fell steadily with increasing stimulus frequency; phase was close to control values at the lowest stimulus frequencies, but a large and variable phase lag developed at higher frequencies. The phase and gain results for rotation in the light 

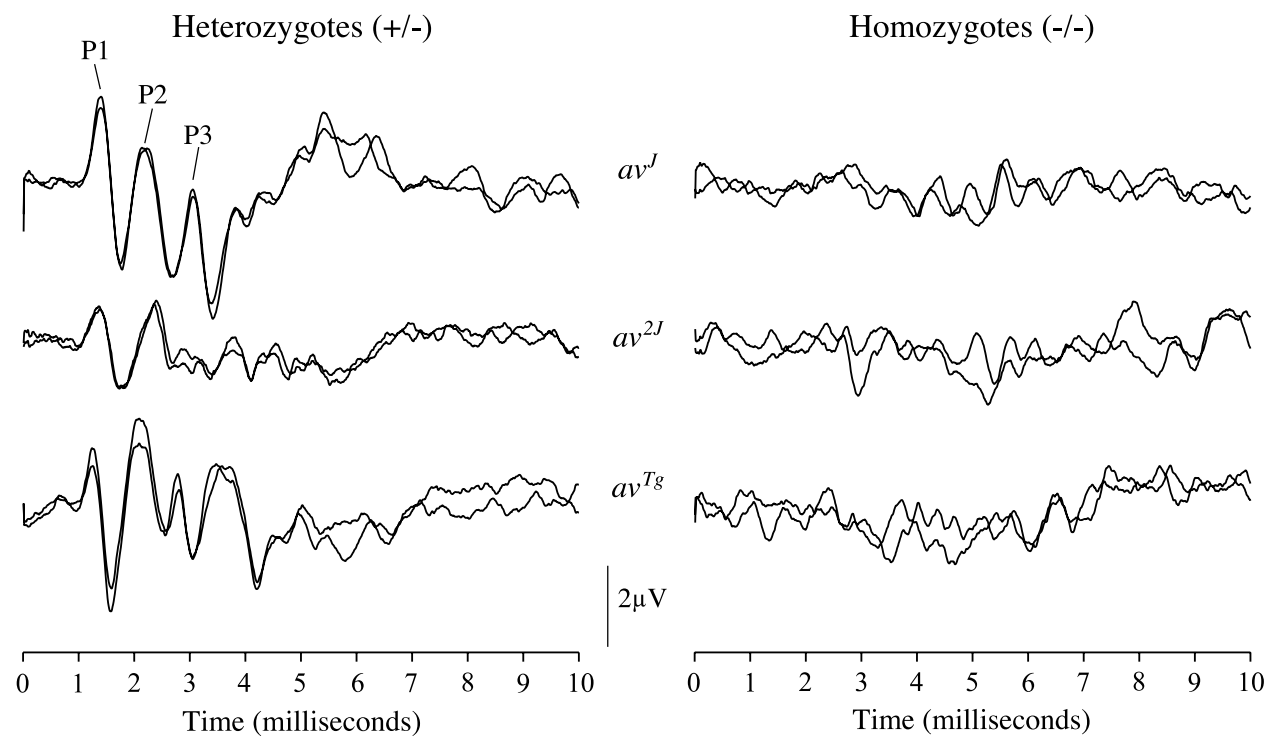

FIG. 5. Representative VsEP waveforms for Pcdh15 ${ }^{\text {av- }}$, Pcdh15 $15^{\mathrm{av}-2 l}$, and Pcdh15 $5^{\mathrm{av}-\mathrm{Tg}}$ heterozygotes $(+/-$ left panel) and homozygotes (-/- right panel). Stimulus intensity used to obtain these traces was $+6 \mathrm{~dB}$ re: $1.0 \mathrm{~g} /$ ms. Total time for each waveform is $10 \mathrm{~ms}$. The first three response peaks are marked in the left panel. are consistent with the $P c d h 15^{a v-3 J}$ response to rotation in the light having arisen entirely from the optokinetic (visual) system.

The aVOR of the mouse has been reported to exhibit nonlinear characteristics, with gain attenuating as stimulus velocity falls (van Alphen et al. 2001). The maximal velocity generated by our sinusoidal stimuli was $39^{\circ} / \mathrm{s}$; to exclude the possibility that we failed to appreciate an aVOR due to having used insufficient stimulus velocities, we also tested animals using pulses of angular velocity that peaked at $105^{\circ} / \mathrm{s}$. The Pcdh $15^{a v-3 J}$ mutants exhibited essentially no response to this stronger stimulus (gain =

$$
a v^{2 J}+/-
$$

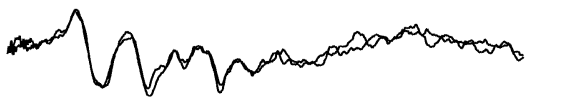

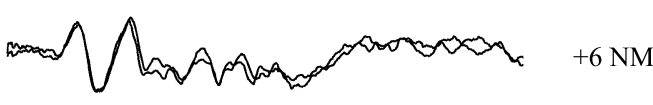

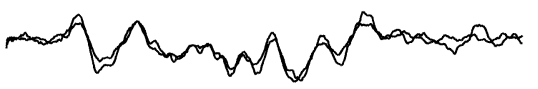

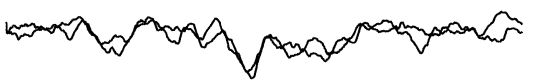

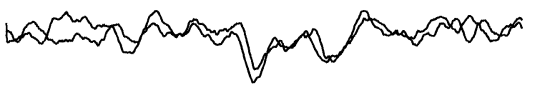

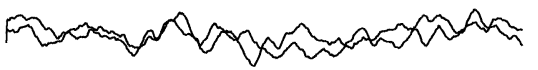

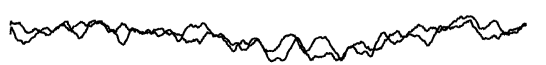

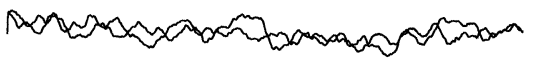

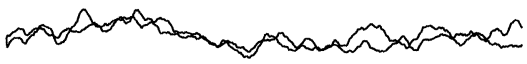

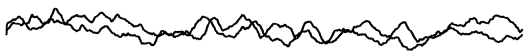

$+6 \mathrm{M}$

$6 \mathrm{NM}$
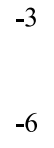

$-9$

$-12$

$-15$

$2 \mu \mathrm{V}$

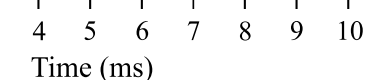

FIG. 6. Intensity functions for one heterozygote (left) and homozygote (right) of the Pcdhl $5^{\text {av-2l }}$ mutation. The homozygote in this case showed circling behavior, normal swimming behavior, and VsEPs were present. VsEP threshold for this homozygote, however, was elevated in comparison to controls. Stimulus intensity is in $\mathrm{dB}$ re: $1.0 \mathrm{~g} / \mathrm{ms}$ and is shown for each trace pair. The top two trace pairs were obtained at $+6 \mathrm{~dB}$ re: $1.0 \mathrm{~g} / \mathrm{ms}$ with (M) and without (NM) an intense wide band forward masker $(100 \mathrm{~dB}$ SPL, 50-50,000 Hz). 


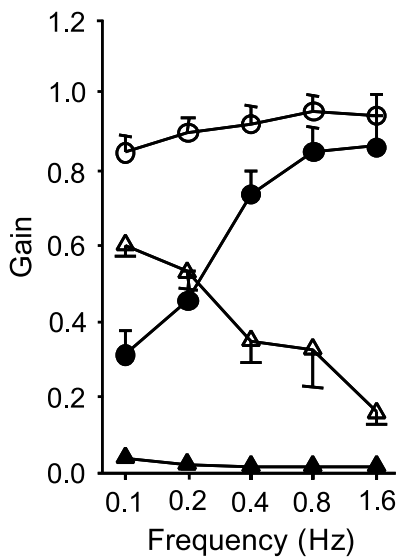

FIG. 7. Plots of angular vestibulo-ocular reflex gain and phase versus stimulus frequency. Control animals and homozygous $P c d h 15^{a v-3 j}$ alleles are plotted as circles and triangles, respectively. Rotation in light and darkness denoted by open and filled symbols, respectively. The phase plot for Pcdh15 av-3j in darkness is omitted

$-0.03 \pm 0.14)$. In comparison, control animals responded robustly (gain $=0.80 \pm 0.10$ ). To exclude the possibility that mutants are deficient in horizontal canal-mediated aVOR alone, we also tested the response to steps with the animal pitched $40^{\circ}$ nose-down from our standard position. Although this angle does not place the vertical semicircular canals in the plane of the stimulus, it still generates at least $64 \%\left(\sin 40^{\circ}\right)$ of the stimulus that would be delivered at the most effective pitch position [based on the assumption that the vertical canals are in their least stimulated (null) orientation when the animal is in the standard position]. Again, Pcdh $15^{a v-3 J}$ mutants exhibited no response (gain $=-0.01 \pm 0.01$ ). Control animals tested at the slightly lesser pitch of $30^{\circ}$ nosedown generated gains of $0.82 \pm 0.04$.

Plots of OKR gain versus drum velocity (i.e., speed tuning curves) are shown in Figure 8. Control animals exhibited optokinetic gains just under 0.8 , declining steadily at stimulus speeds above $5^{\circ} / \mathrm{s}$. The speed tuning curve for $P c d h 15^{a v-3 J}$ mutants was similar in profile, although the attenuation was somewhat sharper. The $P c d h 15^{a v-3 J}$ and control curves differed significantly (repeated-measures ANOVA, $F=19.5, p<$ $0.001)$. The relative preservation of the OKR is in striking contrast to the complete absence of the aVOR and provides indications as to the locus of abnormality in Ames waltzer vestibular system (see Discussion).

\section{Ion channel function in mutant hair cells}

The styryl pyridinium dye FM1-43 or its fixable analog AM1-43 has been used to specifically label normally transducing hair cells in the inner ear. The dye uptake experiment provides a method to visually assay sensory channel function (Gale et al. 2001; Meyers et al. 2003). Freshly isolated utricular macu- because the phase calculation in the absence of a detectable response is spurious. Error bars span 1 standard deviation. The curves for $P c d h 15^{a v-3 J}$ mutants (3j/3J) reflect the absence of an angular vestibulo-ocular reflex and a total reliance on visual inputs to drive compensatory eye movements in the light.

lae, saccular maculae, and semicircular duct cristae from alleles $P c d h 15^{a v-3 J}$ and $P c d h 15^{a v-2 J}$ were tested for AM1-43 dye uptake. Both the presumptive functional null allele $\left(P c d h 15^{a v-3}\right)$ and the less severely affected allele $\left(P c d h 15^{a v-2 J}\right)$ were tested. Specimens from control littermates fluoresced strongly with hair cells filled with dye visible across the sensory epithelium. Specimens from the mutant animals, on the other hand, had very few dye-filled hair cells and a much lower fluorescence overall (Fig. 9). AM1-43 can be incorporated into most living cells via pinocytosis at the cell membrane, and the low-intensity, residual stain seen in tissues from mutant mice is most likely a result of dye uptake via this route. Dye uptake resulting from hair cell transduction, on the other

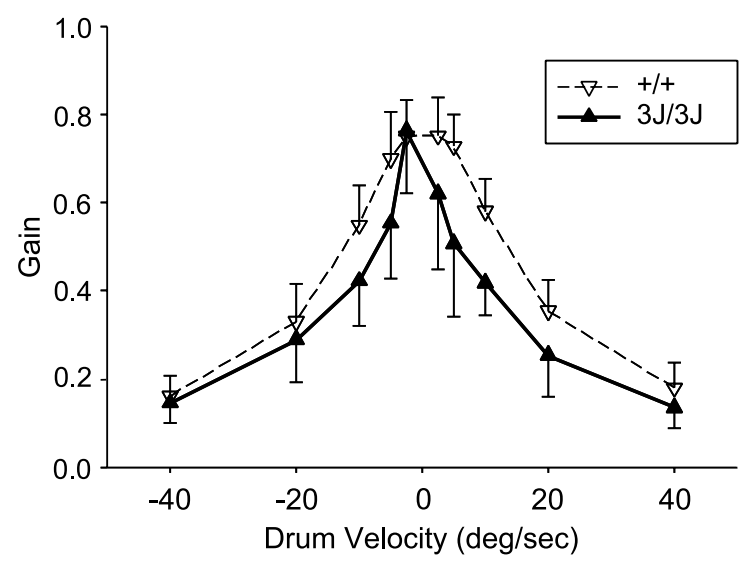

FIG. 8. Plot of horizontal optokinetic reflex gain versus stimulus velocity. Control animals and $P c d h 15^{a v-3 j}$ mutants (3J/3J) are plotted as open and filled triangles, respectively. Error bars span 1 standard deviation. The speed-tuning curve of the $P c d h 15^{\text {av-3l }}$ group is significantly attenuated, but the mutants still generate a robust optokinetic response. 

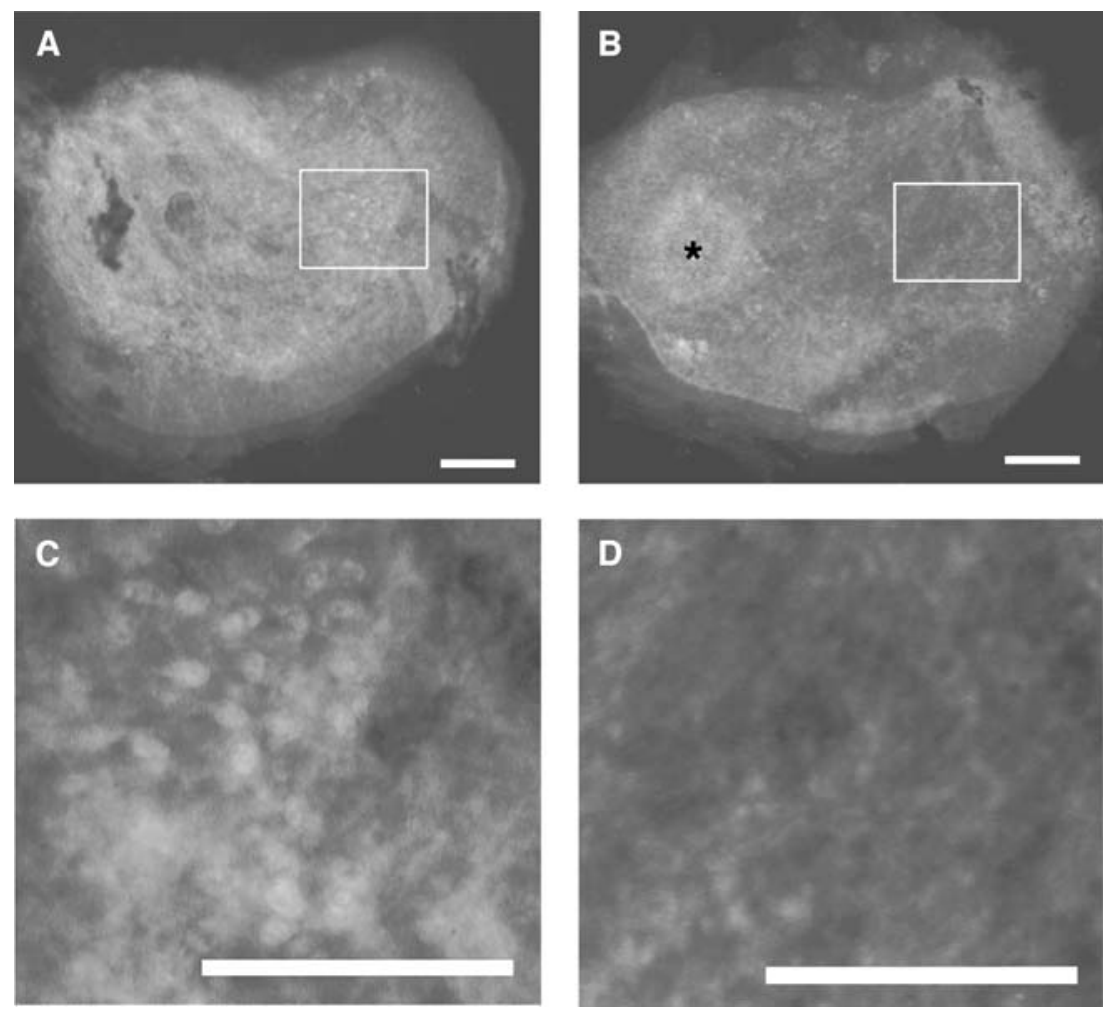

FIG. 9. AM1-43 stained utricular maculae from P10 Pcdh1 $15^{\text {av2s }}$ mutant and control mice. A relatively high fluorescence can be seen across the macular surface in the control mouse (A), compared to the mutant mouse (B). The box in $\mathbf{A}$ outlines an area in which the bodies of sensory cells are in focus. This area is shown at higher magnification in $\mathbf{C}$. $\mathbf{B}$.

The overall fluorescence is significantly lower in the utricular maculae from mutant mice with a reduction in the number of stain-filled hair cells, compared to controls. The asterisk $\left.{ }^{*}\right)$ marks autofluorescence of otoconia that did not come free of the surface at dissection.

The box in $\mathbf{B}$ outlines the area that is shown at higher magnification (D). This area is

in a similar focal plane as B, but lacks whole cell staining of recognizable hair cell bodies. Bar $=100 \mu \mathrm{m}$. hand, typically fills the cell with dye (Fig. 9C), thereby dramatically increasing label intensity and causing the cell to stand out from its neighboring cells. Hair cells were identified by visualization of stereocilia via transmission light microscopy. By fluorescence, dye-filled hair cells were seen much more frequently in the control versus mutant tissue.

\section{DISCUSSION}

Circling behavior in mice is often considered indicative of inner ear vestibular dysfunction. Yet, in the case of Ames waltzer, a structural abnormality consistent with significant vestibular dysfunction has not been identified in young animals, although they display vigorous circling behavior. This raises the question as to whether the observed phenotype might be a result of a central, rather than a peripheral, vestibular defect. The results of this study provide definitive evidence that the circling behavior in the Ames waltzer mutation is associated with severe functional abnormalities of the peripheral vestibular receptor organs. Moreover, our findings suggest that the vestibular dysfunction may be a result of nonfunctional mechanosensory transduction channels in the macular and ampullary receptor cells. This strictly functional vestibular abnormality is in stark contrast to the much more obvious morphological defect seen in the organ of Corti in Ames waltzer mutants.

Several alleles of $a v$ have been identified since the initial discovery of the mutant $a v$ in 1961 (Schaible 1961; Cook and Lane 1993), and the nature of the mutation has been identified in all of the available alleles (Alagramam et al. 2001a; Hampton et al. 2003; Washington et al., in press). In this report, we describe results from a comprehensive study using various methods to characterize vestibular dysfunction in alleles carrying the severe mutation $\left(P c d h 15^{a v-T g}\right.$ or $\left.P c d h 15^{a v-3 J}\right)$ as well as less severe mutations ( $P c d h 15^{a v-2 J}$ or $\left.P c d h 15^{a v-J}\right)$. Unlike other alleles, the Pcdh15 $5^{a v-T g}$ mutation is carried in the $\mathrm{FVB} / \mathrm{N}$ background and therefore is not confounded by problems associated with the B6 background. For most of the studies reported here, we have shown data from $P c d h 15^{a v-T g}$ mice; data from other alleles along with appropriate controls are also presented in this report.

We showed degeneration of the saccular neuroepithelium in older Pcdh15 $5^{a v-T g}$ mutants (P50 or older), which was thought to be consistent with compromised balance function in these animals (Alagramam et al. 1999, 2000). However, based on light microscopic examination, the saccular hair cells from young adults (P15-P30) were indistinguishable from control littermates, although the mutant mice display circling behavior by P10 or earlier. In a study of the three spontaneous mutants of Ames waltzer avJ, -2J, and 
-3J alleles, Raphael et al. (2001) described short, distorted stereocilia on receptor cells of the saccule, utricle, and cristae in animals 15-16 days of age. These observations were based on light microscopic study of phalloidin-stained material and have not been confirmed by scanning electron microscopy. Compared to littermate controls, we did not observe abnormally short, distorted stereocilia on receptor cells of the saccule, utricle, and cristae in mutant animals in our light microscopic or SEM studies.

The changes observed in saccular otoconia at P25 in the functional null allele $P c d h 15^{a v-T g}$ occur before there is any obvious degeneration of sensory cells in the saccular macula, and they may be a result of changes in endolymph composition (very possibly including $\mathrm{pH}$ changes) associated with extensive degeneration of the organ of Corti which occurs by this time. The endolymph of the saccule is in direct communication with that of the cochlear duct via the ductus reuniens. Therefore changes in composition of cochlear endolymph would be readily transmitted to the saccule. Before approximately 25 days of age, the saccular otoconia appear normal, so the degeneration of otoconia is unlikely to contribute to the early circling behavior in these animals.

The VsEP data relating to macular function reveal profound neurophysiologic abnormalities in $P c d h 15^{a v-J}, P c d h 15^{a v-2 J}$, and Pcdh15 $5^{a v-T g}$ alleles of the Ames waltzer mutation. Screening VsEPs obtained previously for the $P c d h 15^{a v-3 J}$ allele also suggested profound functional deficits (Jones et al. 2003). These findings provide definitive evidence of functional deficits in both the saccule and the utricle. Previous studies certainly provided a structural correlate for saccular dysfunction at later ages, but the lack of AM1-43 dye uptake shown here for both the utricle and saccule provides a likely explanation (defective transduction channels) for the profound loss of function in both organs as early as P10. Macular functional deficits could provide one explanation for the balance difficulties observed for this mutation and likely explain the lack of swimming ability described herein.

The fact that one of the $P c d h 15^{a v-2 J}$ mice had observable responses to linear acceleration suggests that vestibular function is not totally compromised in the Pcdh $15^{a v-2 J}$ mutants. Perhaps in this mouse and indeed in the $P c d h 15^{a v-2 J}$ allele in general, the maculae are spared in some cases. Alternatively, one could hypothesize a degenerative condition in which the utricle and saccule might be normal up to the age this animal was tested (38 days old) and then deteriorates thereafter. The other Pcdh $15^{a v-2 J}$ mutants were 42 days old and had absent VsEPs. Further studies are necessary to determine the prevalence of residual gravity receptor function in the $P c d h 15^{a v-2 J}$ allele. $P c d h 15^{a v-2 J}$ carries an in-frame deletion that removes 26 amino acids in the first cadherin domain. Results presented in this report provide evidence that the first cadherin domain of Pcdh15 is important for its function.

VsEPs for the heterozygotes were consistent with previously published data for normal mice (Jones and Jones 1999; Jones et al. 2002). The gradual reduction in VsEP peak latencies across the heterozygote groups appears to be correlated with the age at which the animals were tested. Therefore these data may reflect maturational changes in neural conduction, rather than a pathophysiological difference among the heterozygote alleles.

Eye movement testing revealed that the presumptive null allele $P c d h 15^{a v-3 J}$ lacks a functional angular VOR (aVOR) in darkness. Although the bulk of the stimuli were designed to test horizontal compensatory eye movements, the testing in pitched-down position indicates that the aVOR response related to the vertical semicircular canals was probably absent as well. The eye movement response during rotation in the light must arise entirely as a visual response, and its optokinetic origin is confirmed by the rapid attenuation of the gain and increasing phase lag as stimulus frequency is increased. Direct testing of the visually driven eye movements revealed slightly subnormal optokinetic gains, but the mildness of the deficit is in striking contrast to the complete absence of the aVOR. These results indicate that the Pcdh $15^{a v-3 J}$ mutant is entirely dependent on visual inputs to maintain rotational stability. The visual dependence explains the mutant's gross behavior, in which it alternates periods of relative stability with periods of vigorous circling; once the animal begins to circle, the visual scene drifts rapidly across the retina and the optokinetic system, which performs poorly at higher retinal image velocities, can no longer compensate for the missing vestibular inputs. Thus, when circling, the animal is deprived of the sensory information it requires to reacquire stability within the inertial reference frame.

The relative integrity of the optokinetic response suggests that the lack of the aVOR is attributable to a peripheral abnormality. Single-neuron recordings in a variety of mammalian species have demonstrated that optokinetic and vestibular signals converge at the level of the second-order vestibular neurons (i.e., vestibular nucleus neurons receiving direct inputs from the vestibular nerve) (Hamann and Lannou 1987; Keller and Precht 1978; Precht and Cazin 1979; Stahl and Simpson 1995; Waespe and Henn 1977). The observation that bilateral labyrinthectomy transiently suppresses optokinetic responses has also been interpreted as indicating that secondary vestibular neurons serve as an important conduit for optokinetic signals. Therefore the relative preserva- 
tion of the optokinetic response in $P c d h 15^{a v-3 J}$ argues that the complete absence of aVOR stems from an abnormality upstream from the secondary vestibular neurons, i.e., in the semicircular canals or the primary vestibular neurons. The mild reduction of the optokinetic response raises the possibility that the central pathways are affected by the mutation. The effect may be indirect - a consequence of the loss of vestibular inputs. Mild reductions in optokinetic response can be demonstrated following labyrinthectomy (Collewijn and Grootendorst 1979). In addition, the lack of a normal synergy of optokinetic and vestibular signals during ambulation in light could prevent the mutant from optimally "tuning" its optokinetic system. However, wholly normal optokinetic performance can coexist with complete peripheral vestibular failure (Sun et al. 2001), so our behavioral results do not exclude the possibility that Pcdh15 plays some role in the development or subsequent function of the central pathways.

Obvious morphological defects in the Ames waltzer line are restricted to the neuroepithelium of the saccule, and these defects appear at a time point considerably after the first appearance of circling behavior. We conclude that mutation in Pcdh15 results in early functional abnormality in the saccular hair cells. The utricular macula and the ampullary cristae appear to be anatomically normal in all alleles and do not appear to be affected in animals as old as 1 year. Examination of the neurophysiology of the various vestibular components confirmed the loss of peripheral vestibular function in adult animals. Tests for both the maculae (saccule and utricle) and cristae (the semicircular duct receptors) demonstrated absent responses suggesting that all of the vestibular end organs and/or their associated nerves are not functioning. The lack of function in all the peripheral vestibular receptor organs could be a result of nonfunctional transduction components. Several lines of evidence show that styryl dyes such as FM1-43 (or its fixable analog AM1-43) enter hair cells by passing through the transduction channels near the tips of the stereocilia (Gale et al. 2001; Meyers et al. 2003). Furthermore, entry of FM1-43 is blocked by drugs that block mechanically gated channels (Gale et al. 2001). Therefore specific labeling with these dyes provides a rapid method to assess sensory channel function. The inability of hair cells from Pcdh $15^{a v-3 J}$ and Pcdh15 $5^{a v-2 J}$ mutants to upload the AM1-43 dye is direct evidence that hair cells in these alleles fail to transduce, although the mutation does not appear to have an obvious affect on morphology of the vestibular hair cells.

In conclusion, this study shows that vestibular hair cells in Ames waltzer appear to develop in an anatomically normal manner. Up to the present time, we have not identified a structural defect that might account for the early-onset vestibular dysfunction observed in the various alleles of Ames waltzer. However, it remains possible that future transmission electron microscopic studies may reveal morphologic defects not apparent at the light or scanning electron microscopic levels. Our study shows that Pcdh15 plays an important role in the development of hair cell function, and that the compromised balance function in Ames waltzer mice is caused by lack of input from the vestibular periphery. This study, combined with previous work on cochlear hair cells, indicates that protocadherin 15 has a role in the normal development of structure and function of the inner ear hair cell.

\section{ACKNOWLEDGMENTS}

W. Avniel and M. Hartsock are acknowledged for excellent technical assistance, Jason Meyers for help with the dye uptake experiment, and Usha Ramabadran and Nitin Pagedar for help in preparing this manuscript. This work was supported by American Hearing Research Foundation grant and NIDCD grant RO1 DC05385 to KA and by NIDCD RO1 DC04477 to SMJ and EY13370 to JSS.

\section{REFERENCES}

Ahmed ZM, Riazuddin S, Bernstein SL, Ahmed Z, Khan S, Griffith AJ, Morell RJ, Friedman TB, Riazuddin S, Wilcox ER. Mutations of the protocadherin gene PCDH15 cause Usher syndrome type 1F. Am. J. Hum. Genet. 69:25-34, 2001.

Ahmed ZM, Riazuddin S, Ahmad J, Bernstein SL, Guo Y, Sabar MF, Sieving P, Griffith AJ, Friedman TB, Belyantseva IA, Wilcox ER. PCDH15 is expressed in the neurosensory epithelium of the eye and ear and mutant alleles are responsible for both USH1F and DFNB23. Hum. Mol. Genet. 12:3215-3223, 2003.

Alagramam KN, Kwon HY, Cacheiro NL, Stubbs L, Wright CG, ERWAY LC, WOYCHIK RP. A new mouse insertional mutation that causes sensorineural deafness and vestibular defects. Genetics 152:1691-1699, 1999.

Alagramam KN, Zahorsky-Reeves J, Wright CG, Pawlowski KS, Erway LC, Stubbs L, Wоуснік RP. Neuroepithelial defects of the inner ear in a new allele of the mouse mutation Ames waltzer. Hear. Res. 148:181-191, 2000.

Alagramam KN, Murcia CL, Kwon HY, Pawlowski KS, Wright CG, Wоуснік RP. The mouse Ames waltzer hearing-loss mutant is caused by mutation of PCDH15, a novel protocadherin gene. Nat. Genet. 27:99-102, 2001a.

Alagramam KN, Yuan HJ, Kuehn MH, Murcia Cl, Wayne S, Srisailpathy CRS, Lowry RB, Knaus R, VanLaer L, Bernier FP, et. al. Mutations in the novel protocadherin PCDH15 cause Usher syndrome type 1F. Hum. Mol. Genet. 10:1709-1718, 2001b.

Ben-Yosef T, Ness Sl, Madeo AC, Bar-Lev A, Wolfman JH, Ahmed ZM, Desnick RJ, Willner JP, Avraham KB, Ostrer H, et. al. Brief report - a mutation of PCDH15 among Ashkenazi Jews with the type 1 Usher syndrome. N. Engl. J. Med. 348:1664-1670, 2003.

Collewijn H, Grootendorst A. Adaptation of optokinetic and 
vestibulo-ocular reflexes to modified visual input in the rabbit. In: Granit R, Pompeiano O (eds) Reflex control of posture and movement. Elsevier, New York, pp. 771-781, 1977.

Cook S, Lane P. Re-mutation to Ames waltzer. Mouse Genome 91:554, 1993.

Gale Je, Marcotti W, Kennedy HJ, Kros CJ, Richardson GP. FM1-43 dye behaves as a permeant blocker of the hair-cell mechanotransducer channel. J. Neurosci. 21:7013-7025, 2001.

Hamann KF, LANNOU J. Dynamic characteristics of vestibular nuclear neurons responses to vestibular and optokinetic stimulation during vestibular compensation in the rat. Acta Oto-laryngol., Suppl. 445:1-19, 1987.

Hampton LL, Wright CG, Alagramam KN, Battey JF, Noben-Trauth K. A new spontaneous mutation in the mouse Ames waltzer gene, Pcdh15. Hear. Res. 180:67-75, 2003.

Jones TA, JoNEs SM. Short latency compound action potentials from mammalian gravity receptor organs. Hear. Res. 136:75-85, 1999.

Jones SM, Erway LC, Bergstrom RA, Schimenti JC, Jones TA. Vestibular responses to linear acceleration are absent in otoconia-deficient C57BL/6JEi-het mice. Hear. Res. 135:56-60, 1999.

Jones SM, Subramanian G, Avniel W, Guo Y, Burkard RF, Jones TA. Stimulus and recording variables and their effects on mammalian vestibular evoked potentials. J. Neurosci. Methods 118:23-31, 2002.

Jones SM, Johnson KR, Yu H, Erway LC, Alagramam KN, Jones TA. A survey of vestibular function in mutant mouse strains. Assoc. Res. Otolaryngol. Abst. 2003.

Keller E, Precht W. Persistence of visual response in vestibular nucleus neurons in cerebellectomized cat. Exp. Brain Res. 32:591-594, 1978.

Meyers JR, MacDonald RB, Duggan A, Lenzi D, Standaert DG, Corwin JT, CoREY DP. Lighting up the senses: FM1-43 loading of sensory cells through nonselective ion channels. J. Neurosci. 23:4054-4065, 2003.
Osako S, Hilding DA. Electron microscopic studies of capillary permeability in normal and Ames waltzer deaf mice. Acta Otolaryngol. 71:365-376, 1971.

Precht W, Cazin L. Functional deficits in the optokinetic system of albino rats. Exp. Brain Res. 37:183-186, 1979.

Raphael Y, Kobayashi KN, Dootz GA, Beyer LA, Dolan DF, BurMEISTER M. Severe vestibular and auditory impairment in three alleles of Ames waltzer ( $a v)$ mice. Hear. Res. 151:237-249, 2001.

SCHAIBLE RH. Ames waltzer, av. Mouse News Lett. 24:38, 1961.

Stahl J. Calcium channelopathy mutants and their role in ocular motor research. Ann. N.Y. Acad. Sci. 956:64-74, 2002.

STAHL JS. Eye movements of the murine $\mathrm{P} / \mathrm{Q}$ calcium channel mutant rocker, and the impact of aging. J. Neurophysiol. 91:2066-2078, 2004.

Stahl JS, Simpson JI. Dynamics of rabbit vestibular nucleus neurons and the influence of the flocculus. J. Neurophysiol. 73:1396-1413, 1995.

Stahl JS, van Alphen AM, De Zeeuw CI. A comparison of video and magnetic search coil recordings of mouse eye movements. J. Neurosci. Methods 99:101-110, 2000.

Sun JC, van Alphen AM, Wagenaar M, Huygen P, Hoogenraad CC, Hasson T, Koekkoek SK, Bohne BA, De Zeeuw CI. Origin of vestibular dysfunction in Usher syndrome type 1B. Neurobiol. Dis. 8:69-77, 2001.

van Alphen A, Stahl J, Koekkoek S, Zeeuw CD. The dynamic characteristics of the mouse vestibulo-ocular and optokinetic response. Brain Res. 890:296-305, 2001.

WAESPE W, HenN V. Neuronal activity in the vestibular nuclei of the alert monkey during vestibular and optokinetic stimulation. Exp. Brain Res. 27:523-538, 1977.

Washington JL III, Pitts D, Wright CG, Erway LC, Davis RR, Alagramam KN. Characterization of new allele of Ames waltzer generated by ENU mutagenesis. Hear. Res. (in press).

WiLSON VJ, Jones GM. Mammalian vestibular physiology. Plenum, New York, 1979. 